
\title{
R Reseracth Sulure \\ Radiation shift from triple to quadruple frequency caused by the interaction of terahertz pulses with a nonlinear Kerr medium
}

llya Artser

ITMO University

Maksim Melnik ( $\square$ mmelnik@itmo.ru )

ITMO University

Azat Ismagilov

ITMO University

Mikhail Guselnikov

ITMO University

Anton Tcypkin

ITMO University

Sergei Kozlov

ITMO University

Research Article

Keywords:

Posted Date: February 24th, 2022

DOI: https://doi.org/10.21203/rs.3.rs-1365929/v1

License: (c) (1) This work is licensed under a Creative Commons Attribution 4.0 International License.

Read Full License 


\title{
Radiation shift from triple to quadruple frequency caused by the interaction of terahertz pulses with a nonlinear Kerr medium
}

\author{
Ilya Artser ${ }^{1}$, Maksim Melnik ${ }^{1, *}$, Azat Ismagilov ${ }^{1}$, Mikhail Guselnikov $^{1}$, Anton Tcypkin ${ }^{1}$, and \\ Sergei Kozlov ${ }^{1}$
}

\author{
${ }^{1}$ International Laboratory of Femtosecond Optics and Femtotechnologies, ITMO University, St. Petersburg, Russia \\ *mmelnik@itmo.ru
}

\begin{abstract}
High-intensity optical radiation propagation in a transparent dielectric medium causes the phenomena of self-action of the pulse and the generation of radiation at triple frequencies due to the cubic nonlinearity of the medium. However, usually the quadratic nonlinear effects outshine the cubic ones in the anisotropic nonlinear crystals. In this work, we demonstrate that for certain experimental parameters the nonlinear effect of the third order can be stronger than one of the second order in $\mathrm{MgO}^{\mathrm{LiNbO}} \mathrm{L}_{3}$ crystal for terahertz frequency range. We experimentally and theoretically show that it can lead to the significant modification of the classical phenomenon of radiation generation at triple frequencies in the case when the pulse represents only one complete oscillation of the optical field. In the experiment it was shown that the phenomenon of generation of radiation at triple frequencies with respect to the frequency of the maximum spectral density in a nonlinear medium of the pulse disappears, and it is replaced by the generation of radiation at quadruple frequencies. The analysis demonstrates that this effect is based on the asymmetry of the initial spectrum of such extremely short pulses in terms of the number of oscillations.
\end{abstract}

\section{Introduction}

Research related to the application of terahertz $(\mathrm{THz})$ radiation in various fields of science and industry has been actively carried out over the past 30 years. This is due to the fact that $\mathrm{THz}$ radiation is attractive for such areas as the detection of hidden explosives, diagnostics of food and pharmaceutical products, diagnostics and therapy of socially significant diseases ${ }^{1}$, transmission and receiving of information, including wireless communication devices ${ }^{2,3}$, as well as for various applications in space exploration ${ }^{4}$.

The very first source of electromagnetic $\mathrm{THz}$ radiation was created at the beginning of the last century by the Soviet scientist A.A. Glagoleva-Arkadyeva ${ }^{5}$. In recent years methods have been developed for efficient generation of high-intensity pulsed THz radiation, for example, the method of optical rectification ${ }^{6}$ or generation in plasma ${ }^{7-9}$. Under the excitation of high-intensity $\mathrm{THz}$ radiation, nonlinear effects of media begin to play a significant role ${ }^{10-12}$. It is remarkable that most sources of pulsed terahertz radiation generate pulses containing only a few oscillations of the electrical field ${ }^{13}$. Therefore, nonlinear optics of few-cycle pulses is one of the most perspective and actively developing scientific directions in the field of THz photonics.

Recently, it has been theoretically predicted and experimentally confirmed that the coefficient of the nonlinear refractive index of materials in the terahertz frequency range can be several orders of magnitude higher than its value for the same materials in the visible and near-IR spectral ranges ${ }^{14-16}$. This nonlinearity has low-inertia mechanism, which means that high-speed $\mathrm{THz}$ photonics devices based on nonlinear effects are promising. The analysis of already studied nonlinear effects in the field of pulsed $\mathrm{THz}$ radiation can be found in review papers ${ }^{17-19}$. In addition to the investigation of the features of nonlinear phenomena in the $\mathrm{THz}$ frequency range, the study of new nonlinear materials for this range is actively developing, as well as optimizing existing ones in order to increase the efficiency of nonlinear processes for various applications. There are works that demonstrate modern advances in studies of the generation of the third harmonic of the $\mathrm{THz}$ frequency range in thin graphene layers $^{20,21}$, as well as in doped semiconductors using unipolar THz pulses ${ }^{22}$.

Few-cycle THz pulses are characterized by interesting features of nonlinear optics. For instance, a qualitative change in the nature of the familiar nonlinear effects. For example, it was shown $\mathrm{in}^{23}$ that for pulses with such a small number of oscillations, the classical phenomenon of self-focusing may not be observed even when the critical self-focusing power is many times exceeds. Another classical phenomenon of generation of the second and third harmonics can be significantly modified in pulsed $\mathrm{THz}$ radiation as shown $\mathrm{in}^{24,25}$.

In this study, the effect of the generation of the radiation at quadruple frequencies relative to the frequency of the power spectral density maximum due to interaction of a $\mathrm{THz}$ pulse containing only one full oscillation of the electrical field with a 
cubic nonlinear medium, while the expected radiation at triple frequency is absent for the first time experimentally demonstrated. This effect is believed to be determined by the asymmetry of the spectrum of such a pulse and was demonstrated analytically previously in the study ${ }^{25}$. In addition, based on theoretical calculations, the current work shows that in the THz frequency range, with a certain set of experimental parameters, it is possible to observe an excess of the cubic nonlinearity contribution over the quadratic one for the anisotropic nonlinear crystal, which is usually not observed in the visible frequency range. Due to this, within the framework of the work, it was possible to experimentally observe a pronounced cubic nonlinear effect in a anisotropic $\mathrm{MgO}: \mathrm{LiNbO}_{3}$ crystal. In the work, with the help of refined analytical calculations, the dependence of the position of the dip in the region of triple frequencies, as well as its amplitude relation to the peak in the region of quadruple frequencies, on the nonlinear properties of the material is demonstrated. Obtained analytical results are agreed with experimental data. On the basis of this phenomenon, a method for evaluating the nonlinear refractive index has been developed.

\section{Results}

\section{Experimental results}

The phenomenon of new frequencies generation during the interaction of a high-intensity THz pulse with a nonlinear medium is experimentally observe using a scheme for $\mathrm{THz}$ pulse generation with tilted wavefront in an $\mathrm{MgO}: \mathrm{LiNbO}_{3} \mathrm{crystal}^{26}$. An image of the $\mathrm{THz}$ radiation generation by the optical rectification method is shown in Fig.1(b).

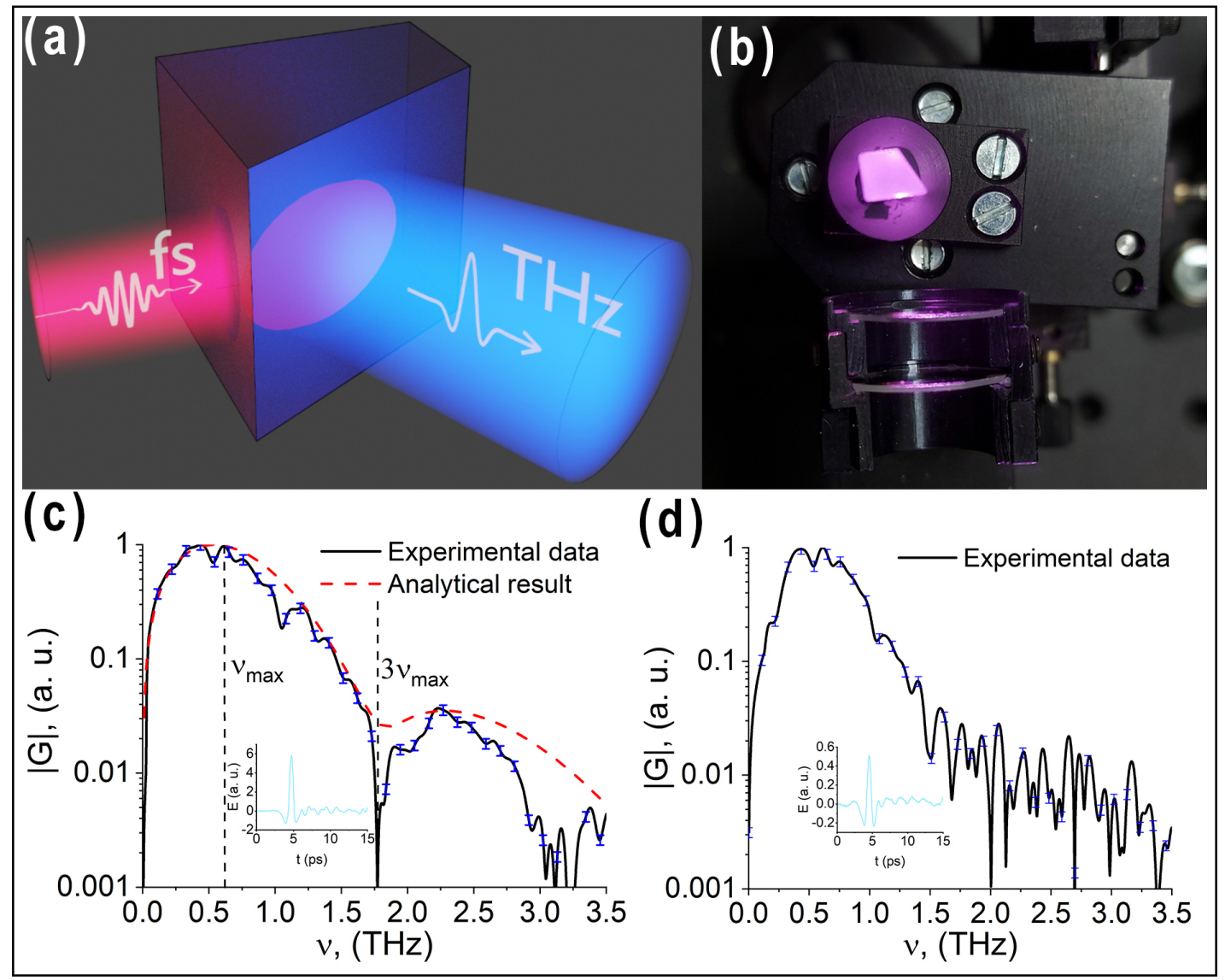

Figure 1. (a) Generation of the THz pulse by optical rectification. (b) Photograph of the generation of a THz pulse by the optical rectification method in $\mathrm{MgO}: \mathrm{LiNbO}_{3}$ crystal. (c) Spectrum of the generated THz field. Black solid line is the experimental data for high pump intensity, red dotted line is the analytical results, blue dashes line shows the error for experimental data. (d) Spectrum of the generated THz field for lower pump intensity.

Thus, a THz pulse with an energy of up to $400 \mathrm{~nJ}$ and a duration of $1 \mathrm{ps}$ is generated. The diameter of the radiation beam at the crystal output is $2 \mathrm{~mm}$, and the radiation intensity reaches $10^{8} \mathrm{~W} / \mathrm{cm}^{2}$.

As seen in Fig.1(c), no generation of radiation at triple frequencies $\left(3 v_{\max }\right)$ is observed in the spectrum of pulsed $\mathrm{THz}$ radiation at the output of the medium during the interaction of the generated $\mathrm{THz}$ field with the crystal, which also has cubic nonlinearity. Moreover, in the THz radiation spectrum instead of the triple frequency, according to the frequency of 
its spectral density maximum, a pronounced dip is observed. In this case, radiation of significant energy is generated at a quadruple frequency, which is not observed for similar experiments in the visible and near-IR frequency ranges. To explain this phenomenon, a theoretical study is carried out.

Figure 1(d) shows the spectrum of a THz pulse generated at a lower pump intensity. As can be seen, such a low intensity is not enough to observe the nonlinear phenomenon of generation of a new frequency. This confirms the fact that the phenomenon of generation of a quadruple frequency instead of the expected triple one which is observed in Fig.1(c), has purely nonlinear origin.

\section{Analytical results}

On the basis of the proposed theoretical model (see Methods), analytical studies of the nonlinear interaction of THz radiation with a medium with quadratic and cubic nonlinearity were carried out, which corresponded to the experiment. The temporal dependence of the $\mathrm{THz}$ pulse field at the input of the optical medium $(2,4)$ is presented on the Fig. 2(a). Figure 2(b) demonstrates spectrum modulus of such $\mathrm{THz}$ pulse as well as the normalized modulus of changes in this spectrum due to quadratic and cubic nonlinearity of the medium $(11,12)$ in the THz frequency range. Figure 2(c) demonstrates the spectrum modulus of the $\mathrm{THz}$ pulse after its propagation in nonlinear medium (9). The maximum of the spectrum modulus corresponds to the frequency of $v_{\max }$ equals to $1 \mathrm{THz}\left(\tau=10^{-12} \mathrm{~s}\right)$. Parameters in the equations was chosen for the lithium niobate crystal used in the experiment: $I=10^{8} \mathrm{~W} / \mathrm{cm}^{2}, N_{0}=5.15, \mathrm{n}_{e l}=2.26, \omega_{0}=5.6 \cdot 10^{12} \mathrm{~s}^{-1}, a_{1}=5.15 \cdot 10^{-8} \mathrm{~cm}, \mathrm{~m}=1.5 \cdot 10^{-22} \mathrm{~g}$, $q=4.8 \cdot 10^{-10} \mathrm{Fr}, \alpha_{T}=1.48 \cdot 10^{-5} \mathrm{~K}^{-1}, S=4.64 \mathrm{~g} / \mathrm{cm}^{3}, N=2.96 \cdot 10^{22} \mathrm{~cm}^{-3}, k_{B}=1.38 \cdot 10^{-16} \mathrm{erg} \cdot \mathrm{K}^{-1}$. All parameters, the dependencies of which are shown in the figures, are normalized to their maximum values.

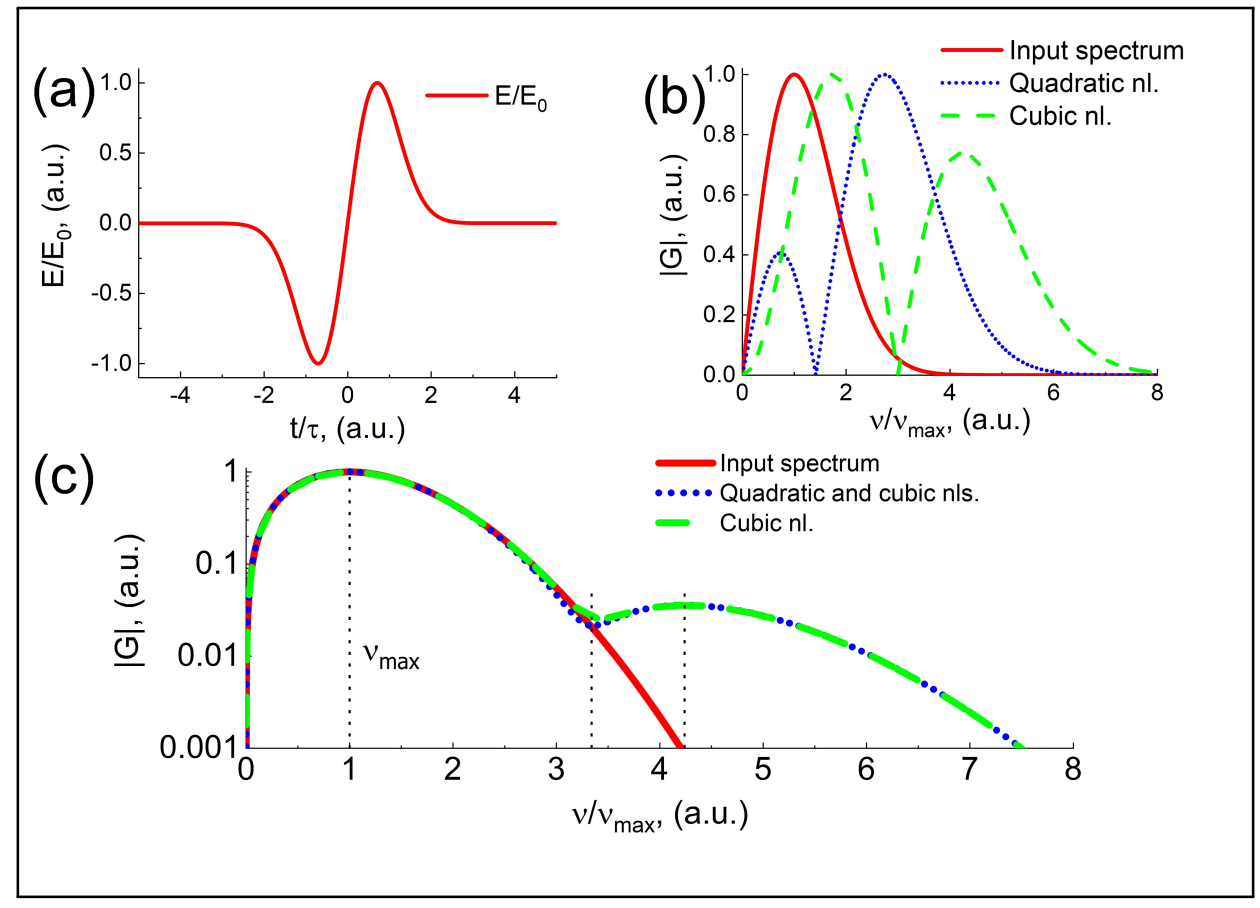

Figure 2. (a) Electric field of a THz single-cycle pulse. (b) The THz pulse spectrum modulus at the input of the medium (red curve), modulus of second-order nonlinear contribution (green dashed curve), modulus of third-order nonlinear contribution (blue dotted curve). (c) Modulus of the THz pulse spectrum at the input of the medium (red curve) and the spectrum of the $\mathrm{THz}$ pulse at the output of the medium with (blue dotted curve) and without (green dashed curve) taking into account the second-order nonlinearity. The position of the maximum frequency $v_{\max }$ of the THz radiation is indicated.

It can be seen from Fig. 2(b) that for a single-cycle pulse, the quadratic nonlinearity leads to the generation of tripled frequencies with respect to the maximum of the single-cycle pulse spectrum at the input of the nonlinear medium instead of doubled ones, and the cubic nonlinearity leads to the generation of quadruple frequencies instead of tripled ones. The first effect was described $\mathrm{in}^{24}$, and the second one $\mathrm{in}^{25}$. As can be seen from Fig. 2(c), at the output of the nonlinear medium in the radiation spectrum in the region of tripled frequency with respect to the maximum spectral density of the initial pulse a pronounced dip is observed (second dotted vertical line), which is corresponds qualitatively to the experimental results (see Fig. $1(\mathrm{c})$ ). Radiation in the medium with quadratic and cubic nonlinearity consideration as well as with only cubic nonlinearity 
consideration is generated at higher frequencies with a maximum in the spectrum shifted to the region of quadruple frequencies of the maximum spectral density of a single-cycle pulse at the input of the nonlinear medium (third dotted vertical line). The analytical expression for the spectrum at the output (9) makes it possible to estimate the position of the extrema of the function by taking the derivative $\partial G / \partial v$ of (9). For example, for the parameters of the medium as in Fig. 2(c), the position of the minimum and maximum in the region of triple and quadruple frequencies, respectively, is 3.34 and 4.24 , and the ratio of their amplitudes is 2.14 . With a decrease in the nonlinearity parameters $\mu_{2}$ and $\mu_{3}$, the ratio between the aforementioned maximum and minimum changes in direct proportion.

It should be noted that Fig. 2(c) demonstrates a negligible contribution of the quadratic nonlinearity with respect to the cubic one, which is not typical for anisotropic crystals in the optical range. To explain this phenomenon, the nonlinear coefficients $\mu_{2}$ and $\mu_{3}$ were calculated from Eq. (3) depending on the radiation intensity (Fig. 3).

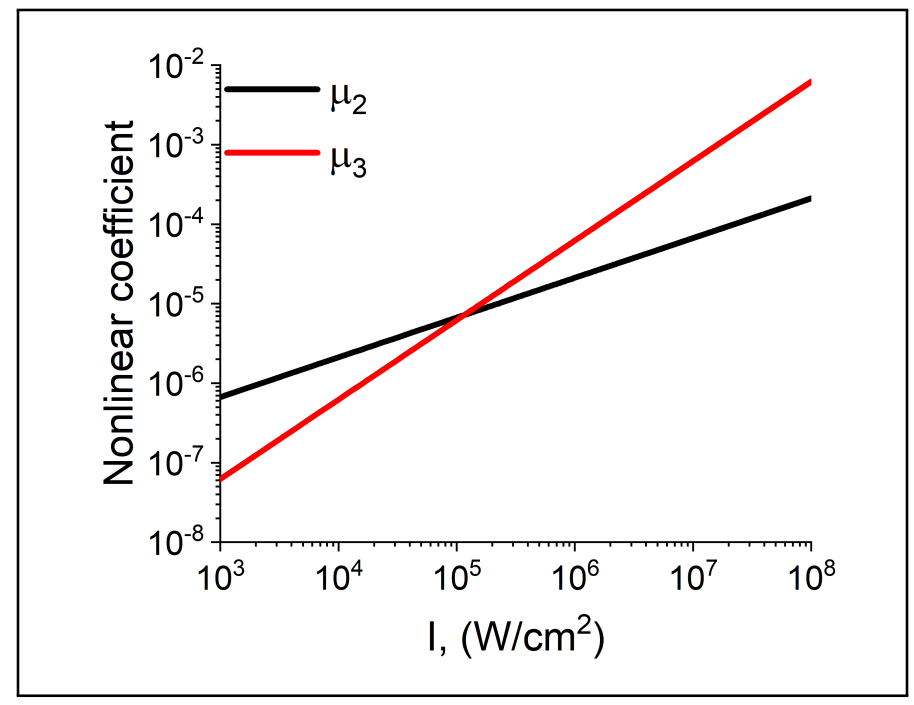

Figure 3. Dependence of quadratic $\left(\mu_{2}\right)$ and cubic $\left(\mu_{3}\right)$ nonlinear coefficients on the THz pulse intensity.

As can be seen from the Fig. 3, there is indeed a range of intensities at which the contribution of the quadratic nonlinearity is higher than that of the cubic one as expected. However, in our experiment the intensity was $10^{8} \mathrm{~W} / \mathrm{cm}^{2}$, and for this intensity the nonlinear coefficients can be calculated as $\chi_{2}=2.7 \cdot 10^{-6} \mathrm{~cm}^{1 / 2} \cdot \mathrm{s} \cdot \mathrm{g}^{-1 / 2}$, so that $\mu_{2}=2.11 \cdot 10^{-4}$, while $\mu_{3}=6.21 \cdot 10^{-3}$. It can be seen that cubic nonlinearity contribution is more than 30 times larger than quadratic one. This makes it possible to observe a cubic nonlinear effect in a anisotropic crystal. This can be explained by the fact that in the THz range, the value of nonlinear refractive index $\mathrm{n}_{2}$ significantly exceeds the value for the visible and IR frequency ranges ${ }^{16}$. For lithium niobate, this excess is 4 orders of magnitude ${ }^{15}$.

In our opinion, such a paradoxical effect of the disappearance of the third harmonic generation in a medium with cubic nonlinearity with the transfer of radiation generation to the region of quadruple frequencies with respect to the maximum spectral density of the optical pulse may be associated with the strongly pronounced asymmetry of the spectrum of a single-cycle pulse.

Figure 2(c) shows the position of the average frequency $\langle v\rangle=\int_{-\infty}^{+\infty} v|G(v)| d v=1.46 v_{\max }$ of the spectrum of a single-cycle pulse and the triple one. The figure demonstrates that the average frequency of the spectrum of a single-cycle pulse is slightly shifted relative to the maximum of the spectral density to the "blue" region, and the position of the maximum in the radiation spectrum generated in a medium with cubic nonlinearity at high frequencies becomes equal to 3.3 from the average frequency.

Nevertheless, with an increase in the number of periods in the pulse, the effect of the disappearance of radiation at tripled frequencies and the appearance of radiation at quadrupled ones disappears. Figure 4 represents solutions (9) considering only cubic nonlinearity for a three-cycle pulse. It can be seen that even with such a small increase in the number of total field oscillations in the initial pulse, its spectrum becomes noticeably more symmetric and the effect of radiation generation at triple frequencies $\left(3 v_{\max }\right)$ practically "returns".

As a result of the above theoretical discussion of the theory, let us compare the results of the experiment and the corresponding theoretical calculations. With the available characteristics of the crystal (which correspond to a crystal thickness of $0.5 \mathrm{~cm}$ ) and the parameters of THz radiation $I=10^{8} \mathrm{~W} / \mathrm{cm}^{2}, \tau=10^{-12} \mathrm{~s}$, the experimental and theoretical curves are in close agreement, which is shown in Fig. 1(c), and occurs for $n_{2}=8 \cdot 10^{-11} \mathrm{~cm}^{2} / W$. This is the measured value of the 


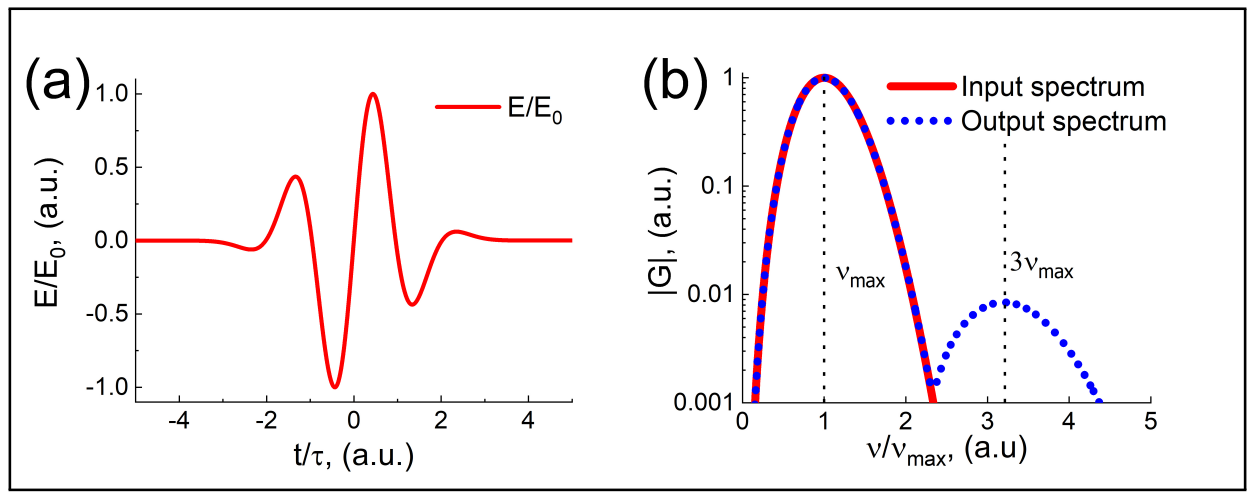

Figure 4. (a) Shape of the pulse with several oscillations electric field. (b) Modulus of the pulse spectrum at the input of the medium (red curve) and the spectrum of the pulse at the output of the medium (blue dotted curve). The position of the maximum frequency $v_{\max }$ of the THz radiation is indicated.

nonlinear refractive index of the $\mathrm{MgO}: \mathrm{LiNbO}_{3}$ crystal in this work. It is very close to the measurement result of the authors of ${ }^{27} n_{2}=4 \cdot 10^{-11} \mathrm{~cm}^{2} / W$. Note that in ${ }^{15}$ the theoretical estimate of this coefficient was $5 \cdot 10^{-11} \mathrm{~cm}^{2} / W$, which is in full agreement with the result of our measurement.

\section{Conclusion}

In the present work, we have shown for the first time experimentally that for the single-cycle $\mathrm{THz}$ pulse the phenomenon of radiation generation at triple frequencies with respect to the frequency of the spectral density maximum in a nonlinear medium disappears, and it is replaced by the generation of radiation at quadruple frequencies. Previously, this phenomenon was described analytically in ${ }^{25}$. In current work, we continued this study and derived analytical formulas that allow us to predict the position of the dip in the region of triple frequencies and the peak in the region of the quadruple frequencies, and also revealed the direct dependence of their ratio on the nonlinear properties of the medium. Furthermore, we reveled,that this phenomenon is related to the strong asymmetry of the spectrum of a single-cycle pulse. In addition, it was found that in the $\mathrm{THz}$ frequency range, with a certain set of experimental parameters, it is possible to observe an excess of the cubic nonlinearity contribution over the quadratic one in a anisotropic lithium niobate crystal, which is usually not observed in the visible frequency range. Due to this fact it was possible to observe a pronounced cubic nonlinear effect in $\mathrm{MgO}: \mathrm{LiNbO}_{3}$ crystal. We used the studied nonlinear effect to measure the nonlinear refractive index of the $\mathrm{MgO}: \mathrm{LiNbO}_{3}$ crystal in the THz spectral range to be $n_{2}=8 \cdot 10^{-11} \mathrm{~cm}^{2} / W$. This result obtained are in complete agreement with other works.

\section{Methods}

\section{Experimental setup}

Experimental setup scheme is shown in Fig.5. Radiation from femtosecond laser system based on a regenerative amplifier (pulse duration is $30 \mathrm{fs}$, central wavelength is $790 \mathrm{~nm}$, pulse energy is $2 \mathrm{~mJ}$, repetition rate is $1 \mathrm{kHz}$ ) is divided into two beams with a beam splitter with 98:2 ratio. Pump radiation goes through optical attenuator into TERA-AX THz generator. Pump radiation after diffraction grating focused on $\mathrm{MgO}: \mathrm{LiNbO}_{3}$ crystal by spherical mirror. Generated $\mathrm{THz}$ radiation collimated by parabolic mirror and goes out of the system. After that THz radiation focused on $1 \mathrm{~mm}$ thick ZnTe crystal by another parabolic mirror for detection. Probe beam after beamsplitter goes to delay line and intersects with $\mathrm{THz}$ pulse at ZnTe crystal and measured by electro-optical setup.

Generation of $\mathrm{THz}$ radiation by the optical rectification method occurs in the entire volume of interaction of the tilted wavefront with the crystal. Thus, THz radiation is generated in the entire volume of crystal as well during the pump radiation propagation through the medium with both quadratic nonlinearity, due to which the THz radiation generation process occurs, and cubic nonlinearity, due to which radiation is expected to be generated at triple frequencies with respect to the THz radiation. A schematic diagram of $\mathrm{THz}$ radiation generation is shown in Fig.1(a). As a result, a diverging $\mathrm{THz}$ radiation beam with a Gaussian profile is formed, directed perpendicular to the crystal cut. Then, THz radiation is collimated using a parabolic mirror with a focal length of $25 \mathrm{~mm}$. The THz field amplitude is measured using a standard electro-optical detection scheme in a 1 mm thick ZnTe crystal. 


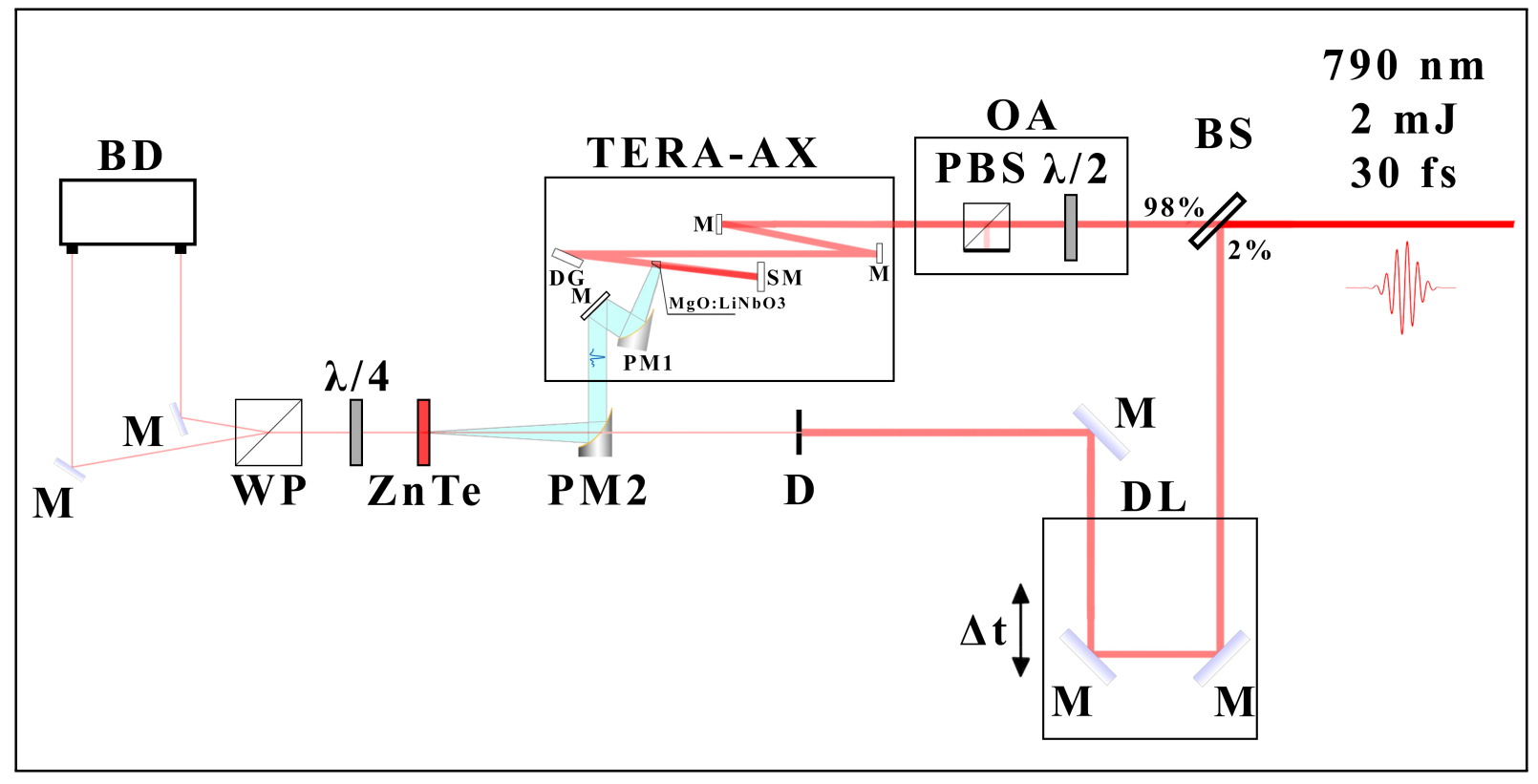

Figure 5. Schematic diagram of the experimental setup. Standard electro optical scheme for detecting the THz field using a 1 $\mathrm{mm}$ thick ZnTe crystal. The THz radiation source is a lithium niobate crystal, pumped by pulsed laser radiation. BS beamsplitter, OA - optical attenuator, PBS - poralizing beamsplitter, $\lambda / 2$ - half-wavelength plate, TERA-AX - terahertz generator, M - mirrors, DG - diffraction grating, SM - spherical mirror, PM - parabolic mirror, DL - delay line, D - diaphragm, ZnTe - $1 \mathrm{~mm}$ thick zinc telluride crystall, $\lambda / 4$ - quarter-wavelength plate, WP - wollaston prism, BD - balanced detector.

\section{Analytical model}

The dynamics of the $\mathrm{THz}$ radiation electric field in a transparent dielectric medium with inertialess quadratic and cubic nonlinearity as well as absorption and amplification consideration can be analyzed on the basis of the equation ${ }^{28}$ :

$$
\frac{\partial E}{\partial z}+\frac{N_{0}}{c} \frac{\partial E}{\partial t}-a \frac{\partial^{3} E}{\partial t^{3}}-(\Gamma-\gamma) E+g_{1} E \frac{\partial E}{\partial t}+g_{2} E^{2} \frac{\partial E}{\partial t}=0,
$$

where $\mathrm{E}$ is electric field, $\mathrm{z}$ is the propagation direction, $\mathrm{t}$ is the time, $c$ is the speed of light in vacuum, $\mathrm{N}_{0}$ and $a$ are the empirical constants characterizing the dependence of the linear refractive index of the medium on the frequency $v$ of the form $n(v)=N_{0}+4 \pi^{2} c a v^{2}, \Gamma$ is the amplification coefficient, $\gamma$ is the absorption coefficient, $g_{1}=\chi_{2} / c$ and $g_{2}=2 n_{2} / c$, are the parameters characterizing the quadratic and cubic nonlinearity of the medium response respectively, $\chi_{2}$ is quadratic susceptibility and $n_{2}$ is the nonlinear refractive index coefficient of the medium (CGS units).

The field at the input of a nonlinear medium (at $z=0$ ) is considered in the form of a single-cycle pulse (see Fig.2(a)) of the form:

$$
E(t, 0)=E_{0} \frac{t}{\tau} e^{-\left(\frac{t}{\tau}\right)^{2}}
$$

where $E_{0}$ is the amplitude of the pulse electric field, $\tau$ is its duration. Figure 1(a) shows that the THz pulse observed in the experiment in the far diffraction zone has one and a half cycle. However, writing the boundary condition in the form of a single-cycle pulse (2), we consider that during the propagation in the crystal, as well as at its output the THz pulse is a single-cycle one and only in the far zone, due to diffraction, it acquires a half-wave ${ }^{29}$. This effect, which is practically not observed for pulses with a large number of oscillations, is clearly pronounced for a single-cycle pulse, which becomes one and a half cycle in the far diffraction zone ${ }^{30}$.

For further analysis, it is useful to rewrite Eq. (1) in normalized variables $\widetilde{E}=E / E_{0}, \widetilde{t}=t / \tau, \widetilde{z}=z / L_{\text {pulse }}$, where $L_{p u l s e}=c \tau / N_{0}$ is the longitudinal size of the THz pulse in the medium:

$$
\frac{\partial \tilde{E}}{\partial \tilde{z}}+\frac{\partial \tilde{E}}{\partial \tilde{t}}-\mu_{0} \frac{\partial^{3} \tilde{E}}{\partial \tilde{t}^{3}}-\mu_{1} \tilde{E}+\mu_{2} \tilde{E} \frac{\partial \tilde{E}}{\partial \tilde{t}}+\mu_{3} \tilde{E}^{2} \frac{\partial \tilde{E}}{\partial \tilde{t}}=0
$$


Here, $\mu_{0}=1 / 2 \cdot \Delta n_{d i s p} / N_{0} . \Delta n_{d i s p}=4 \pi^{2} a c v_{\max }^{2}$ characterizes the change in the refractive index due to dispersion, where $v_{\max }=\sqrt{2} / \tau$ is the the spectral density maximum frequency at the input of the nonlinear medium, $\mu_{1}=\frac{c \tau}{N_{0}}(\Gamma-\gamma)$ characterizes the amplification and absorption of the medium; $\mu_{2}=\frac{\chi_{2} E_{0}}{N_{0}}$ represents the quadratic nonlinearity contribution, where $\chi_{2}=\frac{m \omega_{0}^{2} a_{l} \alpha_{T}}{32 \pi^{2} q N k_{B}}\left(n_{0, v}^{2}-1\right)^{2}, \mu_{3}=4 \Delta n_{n l} / N_{0}, \Delta n_{n l}=1 / 2 n_{2} E_{0}^{2}=n_{2}^{\prime} I$ characterizes the change in the refractive index of the medium due to the cubic nonlinearity, $n_{2}^{\prime}$ is the coefficient of the nonlinear refractive index of the medium (in SI units), $I$ is intensity of THz pulse, $n_{0, v}^{2}=\sqrt{1+N_{0}^{2}-n_{e l}^{2}}$ is the vibrational contribution to the low-frequency refractive index, $n_{e l}$ is the refractive index in the range with nonresonant electronic contribution $(800 \mathrm{~nm}), \omega_{0}$ is the fundamental vibration frequency, $a_{1}$ is the lattice constant, $m$ is the reduced mass of the vibrational mode, $q$ is the effective charge of the chemical bond, $\alpha_{T}$ is the thermal expansion coefficient, $N$ is the number density of vibrational units and $k_{B}$ is the Boltzmann constant.

The normalized boundary condition (2) takes the form

$$
\widetilde{E}(\widetilde{t}, 0)=\widetilde{t} e^{-\widetilde{t}^{2}}
$$

The contribution of dispersion, as is known, leads to a change in the temporal structure of the pulse and does not affect its spectrum. In addition, it can be seen from the experimental data that no dispersive spreading of the pulse is observed; therefore, the dispersive contribution can be neglected.

The dependence of the absorption spectrum in the studied spectral range has no characteristic features ${ }^{31}$. The result of taking into account the absorption coefficient leads to a monotonic decrease in the power spectrum of the output $\mathrm{THz}$ radiation. In this regard, absorption can be neglected in calculations.

For ease of taking into account the amplification of the THz pulse, in the calculations we consider the case when the energy of the pulse interacting with the medium corresponds to the energy of the pulse at the output from the medium. Therefore, in the propagation dynamics equation, the amplification term can also be omitted.

Thus, for an analytical calculation of the dynamics of a THz pulse during propagation, one can focus on the last two terms of Eq. (3). Assuming the values $\mu_{2}$ and $\mu_{3}$ are small, the solution to Eq. (3) can be found in the form of a series:

$$
\tilde{E}(\tilde{t}, \tilde{z})=\tilde{E}^{0}(\tilde{t}, \tilde{z})+\mu_{2} \tilde{E}^{(1, \mathrm{n} 12)}(\tilde{t}, \tilde{z})+\mu_{3} \tilde{E}^{(1, \mathrm{n} 13)}(\tilde{t}, \tilde{z})
$$

It is easy to show that with boundary conditions (2), the terms in such a solution take the form

$$
\begin{aligned}
& \widetilde{E}^{(0)}(\widetilde{t}, \widetilde{z})=e^{-(\widetilde{t}-\widetilde{z})^{2}}(\widetilde{t}-\widetilde{z}) \\
& \tilde{E}^{(1, \mathrm{n} 12)}(\tilde{t}, \tilde{z})=\widetilde{z}(\widetilde{t}-\widetilde{z})\left(2(\widetilde{t}-\widetilde{z})^{2}-1\right) e^{-2(\widetilde{t}-\widetilde{z})^{2}} \\
& \widetilde{E}^{(1, n l 3)}(\widetilde{t}, \widetilde{z})=\widetilde{z}(\widetilde{t}-\widetilde{z})^{2}\left(2(\widetilde{t}-\widetilde{z})^{2}-1\right) e^{-3(\widetilde{t}-\widetilde{z})^{2}}
\end{aligned}
$$

Here, $\widetilde{E}^{(0)}$ is the solution of equation (3) for the "zero" approximation, i.e. without taking into account the nonlinearity of the medium, $\widetilde{E}^{(1, n l 2)}$ and and $\widetilde{E}^{(1, n l 3)}$ are the change in the shape of the pulse, associated with quadratic and cubic nonlinearity respectively.

Accordingly, the field (5) spectrum $G(\widetilde{v}, \widetilde{z})=1 / \sqrt{2 \pi} \int_{-\infty}^{+\infty} \widetilde{E}(\widetilde{t}, \widetilde{z}) \cdot e^{i \widetilde{v t}} d \widetilde{t}$ where $\widetilde{v}=v \tau$ can be represented as

$$
\begin{aligned}
& \widetilde{G}(\widetilde{v}, \widetilde{z})=\widetilde{G}^{(0)}(\widetilde{v}, \widetilde{z})+\mu_{2} \widetilde{G}^{(1, n l 2)}(\widetilde{v}, \widetilde{z})+\mu_{3} \widetilde{G}^{(1, n l 3)}(\widetilde{v}, \widetilde{z}) \\
& \widetilde{G}(0)(\widetilde{v}, \widetilde{z})=\frac{i e^{i \tilde{z} \tilde{v}-\widetilde{v}^{2} / 4 \widetilde{v}}}{2 \sqrt{2}} \\
& G^{(1, n 12)}(\tilde{v}, \tilde{z})=-\frac{i \tilde{v} \tilde{z}\left(\widetilde{v}^{2}-4\right) e^{-\tilde{v}^{2} / 8+i \tilde{v} \tilde{z}}}{64}
\end{aligned}
$$




$$
\widetilde{G}^{(1, n l 3)}(\widetilde{v}, \widetilde{z})=\frac{\tilde{z} \tilde{v}^{2}\left(\widetilde{v}^{2}-18\right) e^{-\widetilde{v}^{2} / 12+i \tilde{v} \tilde{z}}}{648 \sqrt{6}}
$$

\section{References}

1. Zhang, X.-C. \& Xu, J. Introduction to THz wave photonics, vol. 29 (Springer, 2010).

2. Grachev, Y. V. et al. Wireless data transmission method using pulsed thz sliced spectral supercontinuum. IEEE Photonics Technol. Lett. 30, 103-106 (2017).

3. Liu, X. et al. Formation of gigahertz pulse train by chirped terahertz pulses interference. Sci. Reports 10, 1-7 (2020).

4. Siles, G. A., Riera, J. M. \& Garcia-del Pino, P. Atmospheric attenuation in wireless communication systems at millimeter and thz frequencies [wireless corner]. IEEE Antennas Propag. Mag. 57, 48-61 (2015).

5. Glagolewa-Arkadiewa, A. Short electromagnetic waves of wave-length up to 82 microns. Nature 113, 640-640 (1924).

6. Bass, M., Franken, P., Ward, J. \& Weinreich, G. Optical rectification. Phys. Rev. Lett. 9, 446 (1962).

7. Ponomareva, E. A., Stumpf, S. A., Tcypkin, A. N. \& Kozlov, S. A. Impact of laser-ionized liquid nonlinear characteristics on the efficiency of terahertz wave generation. Opt. letters 44, 5485-5488 (2019).

8. Koulouklidis, A. D. et al. Observation of extremely efficient terahertz generation from mid-infrared two-color laser filaments. Nat. communications 11, 1-8 (2020).

9. Ponomareva, E. A. et al. Varying pre-plasma properties to boost terahertz wave generation in liquids. Commun. Phys. 4, $1-7$ (2021).

10. Tcypkin, A. N. et al. High kerr nonlinearity of water in thz spectral range. Opt. express 27, 10419-10425 (2019).

11. Novelli, F. et al. Strong anisotropy in liquid water upon librational excitation using terahertz laser fields. The J. Phys. Chem. B 124, 4989-5001 (2020).

12. Novelli, F. et al. Nonlinear terahertz transmission by liquid water at 1 thz. Appl. Sci. 10, 5290 (2020).

13. Lee, Y.-S. Principles of terahertz science and technology, vol. 170 (Springer Science \& Business Media, 2009).

14. Dolgaleva, K., Materikina, D. V., Boyd, R. W. \& Kozlov, S. A. Prediction of an extremely large nonlinear refractive index for crystals at terahertz frequencies. Phys. Rev. A 92, 023809 (2015).

15. Zhukova, M., Melnik, M., Vorontsova, I., Tcypkin, A. \& Kozlov, S. Estimations of low-inertia cubic nonlinearity featured by electro-optical crystals in the thz range. Photonics 7, 98 (2020).

16. Tcypkin, A. et al. Giant third-order nonlinear response of liquids at terahertz frequencies. Phys. Rev. Appl. 15, 054009 (2021).

17. Nicoletti, D. \& Cavalleri, A. Nonlinear light-matter interaction at terahertz frequencies. Adv. Opt. Photonics 8, 401-464 (2016).

18. Buccheri, F., Huang, P. \& Zhang, X.-C. Generation and detection of pulsed terahertz waves in gas: from elongated plasmas to microplasmas. Front. Optoelectronics 11, 209-244 (2018).

19. Lin, S., Yu, S. \& Talbayev, D. Measurement of quadratic terahertz optical nonlinearities using second-harmonic lock-in detection. Phys. Rev. Appl. 10, 044007 (2018).

20. Al-Naib, I., Poschmann, M. \& Dignam, M. M. Optimizing third-harmonic generation at terahertz frequencies in graphene. Phys. Rev. B 91, 205407 (2015).

21. Hafez, H. A. et al. Extremely efficient terahertz high-harmonic generation in graphene by hot dirac fermions. Nature 561, 507-511 (2018).

22. Chai, X. et al. Subcycle terahertz nonlinear optics. Phys. review letters 121, 143901 (2018).

23. Kozlov, S. A., Drozdov, A. A., Choudhary, S., Kniazev, M. A. \& Boyd, R. W. Suppression of self-focusing for few-cycle pulses. JOSA B 36, G68-G77 (2019).

24. Sazonov, S. V. Optical rectification and generation of harmonics under condition of propagation of few-cycle pulses in the birefringent medium with asymmetric molecules. J. Russ. Laser Res. 39, 252-262 (2018). 
25. Drozdov, A. A., Kozlov, S. A., Sukhorukov, A. A. \& Kivshar, Y. S. Self-phase modulation and frequency generation with few-cycle optical pulses in nonlinear dispersive media. Phys. Rev. A 86, 053822 (2012).

26. Hebling, J., Yeh, K.-L., Hoffmann, M. C., Bartal, B. \& Nelson, K. A. Generation of high-power terahertz pulses by tilted-pulse-front excitation and their application possibilities. JOSA B 25, B6-B19 (2008).

27. Korpa, C., Tóth, G. \& Hebling, J. Interplay of diffraction and nonlinear effects in the propagation of ultrashort pulses. $J$. Phys. B: At. Mol. Opt. Phys. 49, 035401 (2016).

28. Kozlov, S. A. \& Samartsev, V. V. Fundamentals of femtosecond optics (Elsevier, 2013).

29. Goodman, J. W. Introduction to Fourier optics (Roberts and Company Publishers, 2005).

30. Ezerskaya, A. A., Ivanov, D. V., Kozlov, S. A. \& Kivshar, Y. S. Spectral approach in the analysis of pulsed terahertz radiation. J. Infrared, Millimeter, Terahertz Waves 33, 926-942 (2012).

31. Buzády, A. et al. Temperature-dependent terahertz time-domain spectroscopy study of mg-doped stoichiometric lithium niobate. Opt. Mater. Express 10, 998-1006 (2020).

\section{Acknowledgements (not compulsory)}

This research was funded by RSF project 22-22-20059.

\section{Author contributions statement}

A.T. conceived the experiment. A.I. and A.T. conducted the experiment. A.I. and M.M. analysed the experimental results. I.A.and S.K. developed the theoretical model. I.A. and M.G. obtained analytical results and describe them. All authors reviewed the manuscript.

\section{Additional information}

Competing interests: The authors declare that they have no competing interests.

Data availability: All data generated or analyzed during this study are included in this published article and its supplementary information files. 